\title{
Challenges in Differentiating Between Solitary Rectal Ulcer Syndrome and Inflammatory Bowel Disease in the Pediatric Population
}

\author{
Shouli Tung ${ }^{\mathrm{a}}$, Paige Richards ${ }^{\mathrm{a}}$, Gabriele Hunter ${ }^{\mathrm{b}}$, John Tung ${ }^{\mathrm{a}, \mathrm{c}}$
}

\begin{abstract}
Solitary rectal ulcer syndrome (SRUS) is a benign rectal disease that is rare in pediatric populations due to its underdiagnosis and misdiagnosis in children. It is often misdiagnosed as malignancy, Crohn's disease, and ulcerative colitis due to its wide and varying clinical presentations. Both SRUS and inflammatory bowel disease (IBD) can present with rectal bleeding, constipation, diarrhea, and abdominal pain. Furthermore, macroscopic ulcers and inflammation can be seen in both diseases, making it difficult to diagnose without a biopsy. We present two cases in the pediatric population whose diagnoses of SRUS were delayed because the symptoms and macroscopic findings initially supported the differential diagnosis of IBD. These cases emphasize the difficulty and importance of differentiating between IBD and SRUS, and should encourage practitioners to include this differential diagnosis earlier on to improve diagnostic accuracy and begin implementing effective treatment. This can eventually decrease overall treatment time, unnecessary surgeries and diagnostic testing, and increase the emotional reassurance of the benign nature of the disease.
\end{abstract}

Keywords: Solitary rectal ulcer syndrome; Inflammatory bowel disease; Rectal bleeding; Children; Diagnosis; Treatment

\section{Introduction}

Solitary rectal ulcer syndrome (SRUS) is a syndrome that most often presents with rectal bleeding, pelvic fullness, and straining during defecation. Other less common symptoms include mucus discharge, tenesmus, rectal pain, and incontinence. It seems to affect both genders equally, but has a slight predominance towards females. Its incidence has also been associated

Manuscript submitted November 29, 2020, accepted April 2, 2021

Published online September 30, 2021

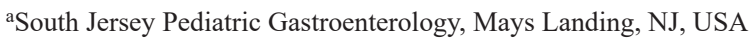

${ }^{b}$ Nemours/Alfred I. DuPont Hospital for Children, Wilmington, DE, USA

${ }^{\mathrm{c} C o r r e s p o n d i n g ~ A u t h o r: ~ J o h n ~ T u n g, ~ S o u t h ~ J e r s e y ~ P e d i a t r i c ~ G a s t r o e n t e r o l o g y, ~}$

Mays Landing, NJ, USA. Email: tungj@yahoo.com

doi: https://doi.org/10.14740/ijcp422 with rectal prolapse [1]. Martin et al found that solitary ulceration was only present $35 \%$ of the time, multiple "solitary" ulcers were present $22 \%$ of the time, broad-based polypoid lesions were found in $25 \%$ of cases, and patchy granular hyperemic rectal mucosa was found in $18 \%$ of the cases, explaining that the name "solitary rectal ulcer syndrome" is a misnomer [2]. It is a well-known syndrome in adults, but thought to be rarer in children due to misdiagnosis or underdiagnosis. Even after endoscopy, SRUS is often initially misdiagnosed as Crohn's disease, mucosal ulcerative colitis, and rectal cancer, especially after polypoid ulcers are found [3]. Diagnosis is made based on clinical symptoms, colonoscopy findings, and histologic finding of fibromuscular obliteration of the lamina propria. Inflammatory bowel disease (IBD) and tumors are differentiated from SRUS with histopathology findings: SRUS shows lamina propria scarring and mild inflammatory infiltrate and muscular hyperplasia [4]. Both pediatric and adult literature of SRUS strive to follow the same stepwise pattern of treatments that begin with conservative methods, such as patient education in behavioral and diet changes, then to topical therapy such as sucralfate enema, corticosteroids, and sulfasalazine, and at last resort, surgical intervention [5]. Pediatric treatment for SRUS specifically stresses patient education and reassurance of the benign nature of the disease to ensure mental well-being and a proper understanding of how to navigate the disease long term [6].

We present two cases of solitary rectal ulcer in two pediatric patients, presenting with symptoms very similar to that of IBD. However, upon further history taking, diagnostic testing, and laboratory results, the diagnosis of SRUS was made with a confirmatory histologic biopsy.

\section{Case Reports}

\section{Case 1}

A 4-year-old male with no significant past medical history presented with postprandial abdominal pain, reduced appetite and intermittent vomiting. He was born in Egypt and had recently moved to the USA 2 years prior. They had city water, and there was a smoker in the household. The only significant family medical history was hypercholesterolemia in his mother. He had no known drug allergies and was not on any medications. 
Review of systems was negative other than the presenting gastrointestinal symptoms, and there were no abnormal physical exam findings. He was found to have Helicobacter pylori $(H$. pylori) immunoglobulin $\mathrm{G}$ (IgG) level of 1.6, and endoscopy also confirmed $H$. pylori gastritis with erosions. He completed 2 weeks of oral metronidazole $150 \mathrm{mg}$ twice daily (BID), oral amoxicillin $150 \mathrm{mg}$ BID, and oral omeprazole $10 \mathrm{mg}$ BID. Treatment with the same course was repeated an additional two times until his $H$. pylori antigen was negative.

Two years later, he developed painless rectal bleeding, constipation, and straining with defecation. Physical exam did not show any abnormal findings. Fecal occult blood test was negative and stool studies for routine pathogens including Clostridium difficile toxin were also negative. Bleeding was attributed to possible small internal fissure, so fiber and mineral oil were recommended daily for 2 months. After 1-month follow-up, he reported increasing amounts of frank blood in his stool without any abdominal pain, so a colonoscopy was scheduled. Colonoscopy noted aphthous ulcers in his rectum and biopsies from the terminal ileum to the rectum showed changes of chronic inflammation in the rectum. He was started on mesalamine $400 \mathrm{mg}$ thrice daily (TID), ferrex $150 \mathrm{mg}$ once daily (QD), and folic acid $1 \mathrm{mg}$ QD after colonoscopy suggested a possible diagnosis of ulcerative colitis. Labs including complete blood count (CBC), complete metabolic panel (CMP), erythrocyte sedimentation rate (ESR), C-reactive protein (CRP), urinalysis, anti-streptolysin $\mathrm{O}$, Lyme titers, rheumatoid arthritis factor, stool lactoferrin, and iron studies were all found to be normal. The only abnormal findings on his other labs were positive anti-nuclear antibodies (ANAs) and total cholesterol of 211 with elevated low-density lipoprotein (LDL) at 118, showing some degree of familial hypercholesterolemia. At the 3-month follow-up, his symptoms resolved and he continued on maintenance therapy of his mesalamine $400 \mathrm{mg}$ TID, ferrex $150 \mathrm{mg}$ QD, and folic acid $1 \mathrm{mg}$ QD. For the next 5 years, he was stable on his medication regimen, continued to have very mild symptoms, was gaining weight appropriately, and his follow-up colonoscopies were normal.

However, 1 year later, he began complaining of distension and borborygmi, so labs were ordered to ensure that he was still in remission of his ulcerative colitis. He was also started on oral metronidazole $250 \mathrm{mg}$ BID for 7 days and a course of probiotics for 14 days after the course of metronidazole. At the 6-month follow-up, he continued to complain of bloating, nausea and discomfort. He also began seeing clots in his stool. Labs showed a slightly elevated ESR at 17, but normal CRP, serum ferritin, and fecal calprotectin. Therefore, he was started on oral prednisone $30 \mathrm{mg}$ daily for suspicion of an ulcerative colitis flare, and a colonoscopy was performed which showed a solitary rectal ulcer (Fig. 1). Histology also confirmed a solitary ulcer with necrotic exudate and acute and chronic inflammatory cells with atrophic, reactive mucosal glands confirming the diagnosis of SRUS (Fig. 2). Furthermore, no granulomas or dysplasia were appreciated. The transverse colon, cecum, and ileum were all normal. At this time, labs showed elevated thyroid stimulating hormone at $7.5 \mathrm{mIU} / \mathrm{L}$, normal free T4 at $1.17 \mathrm{ng} / \mathrm{dL}$, positive thyroid peroxidase antibodies and thyroglobulin antibodies. Mesalamine suppository $1 \mathrm{~g}$ QD
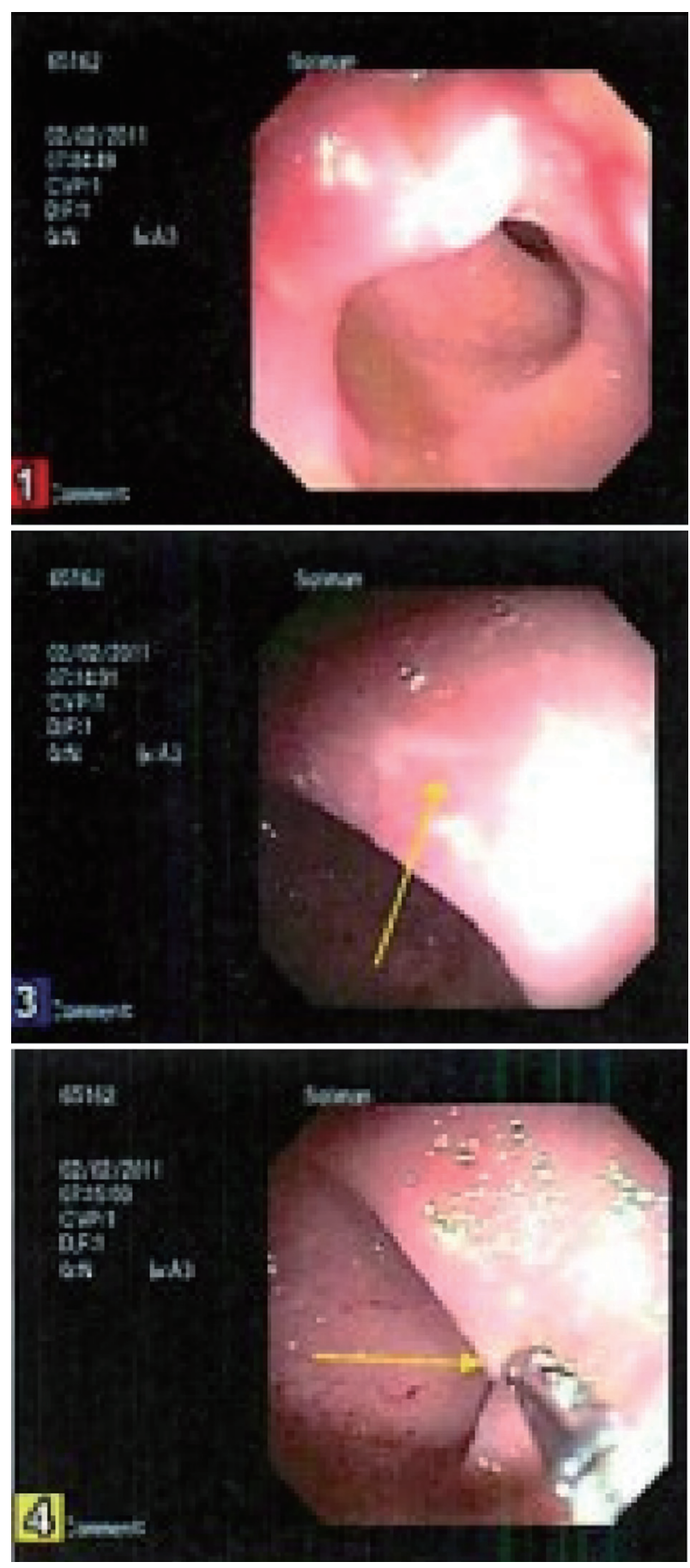

Figure 1. Aphthous ulcers in the rectum.

and oral omeprazole $20 \mathrm{mg}$ QD were added to his medication regimen of oral mesalamine $0.375 \mathrm{~g}$ BID and folic acid $1 \mathrm{mg}$ QD. He was also started on oral docusate $200 \mathrm{mg}$ daily for 4 months and advised to increase his fiber intake for regular bowel movements. He improved on the new regimen, and was able to discontinue the mesalamine suppository and omeprazole after 4 months. At the 1-year follow-up, his symptoms had resolved and he has been stable on his medication regimen of 


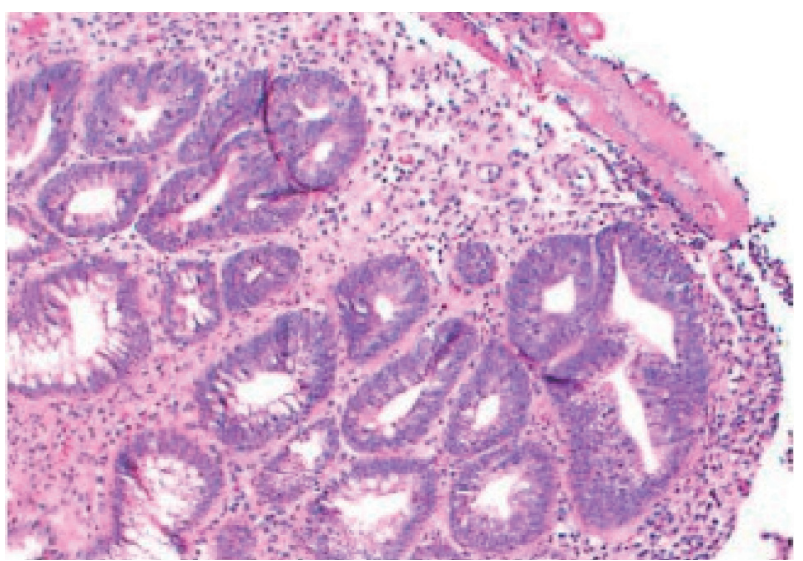

Figure 2. Biopsy of the solitary rectal ulcer. There is destruction of the mucosa with a fibrinopurulent exudate seen on the surface and proliferation of reactive capillaries seen beneath, with acute and chronic inflammation seen in the stroma. Colonic mucosa shows distorted architecture with hyperplastic glands, crypt enlargement, uneven spacing and branching. Fibrosis is seen within the stroma. Mild acute and chronic inflammation is present.

oral mesalamine $0.375 \mathrm{~g}$ BID and folic acid $1 \mathrm{mg}$ QD.

\section{Case 2}

A 15-year-old male with a past medical history of chronic constipation and stool incontinence presented to the hospital with fatigue, weakness, dizziness, pallor, headache, and frequent episodes of diarrhea. He had a weight loss of 30 pounds over the last 18 months which he attributed to a very poor appetite. He denied any vomiting, rectal bleeding, hematemesis, or abdominal pain. There were no recent sick contacts. Family medical history was only positive for diabetes mellitus in his father. On physical exam, he appeared ill, thin and pale, but all other systems were negative. Rectal exam was normal without any perianal lesions or frank blood. Neurologic exam showed that he was alert and oriented, with cranial nerves II to XII intact, no unilateral weakness, and good tone and strength throughout. At the time of admission, he was afebrile with a heart rate of 124 beats per minute, blood pressure of 126/64 $\mathrm{mm} \mathrm{Hg}$, respiratory rate of 20, and oxygen saturation of $99 \%$. On his $\mathrm{CBC}$, he was found to have hemoglobin $6.6 \mathrm{~g} / \mathrm{dL}$ requiring two iron transfusions. Labs showed normal calprotectin, anti-gliadin IgA and IgG levels, and negative stool cultures for Clostridium difficile, Campylobacter, Salmonella, Vibrio, and Yersinia. CRP was slightly elevated at $2 \mathrm{mg} / \mathrm{L}$ and ESR was elevated at $48 \mathrm{~mm} / \mathrm{h}$ (Table 1). An abdominal ultrasound showed some biliary sludge with no other abnormal findings. Colonoscopy only showed rectal edema, no obvious ulcerations, with some exudate (Fig. 3). However, the pathology report showed a severely active chronic colitis with ulcerative mucosa with numerous neutrophils and granulation tissue formation in his rectum. Magnetic resonance enterography (MRE) showed bowel inflammation and wall thickening of the rectum as well as fibrofatty plaque formation and edema around the rectum. His differential diagnoses included IBD, short segment Hirschsprung's disease, and non-infective gastroenteritis and colitis. He was started on oral ferrous sulfate $325 \mathrm{mg}$ BID and instructed to follow-up in 1 week.

At his 1-week follow-up visit, he was asymptomatic. On physical exam, he appeared pale but the rest of the exam was normal. Rectal exam showed no perianal lesions and internal rectal exam showed some dark stool that did not resemble blood. However, his hemoccult was positive, so he was started on mesalamine suppository $1 \mathrm{~g}$ QD until his next follow-up visit. Because of his normal inflammatory markers and normal fecal calprotectin levels during the hospital admission, treatment for IBD was held. At his 1-month follow-up visit, his hemoccult was negative and subsequent labs, endoscopy, and colonoscopy were repeated. Upper endoscopy showed an incidental finding of eosinophilic esophagitis (EOE) as well as mild chronic inflammation in the gastric antrum. Colonoscopy showed focal acute and chronic inflammation with erosions and ulcers without granulomas and dysplasia. Biopsies taken from the transverse colon, cecum, and terminal ileum were all normal. The diagnosis of SRUS was made due to his colonoscopy results and the thickening of his rectum previously seen on MRE, as well as the inflammation seen on magnetic resonance imaging (MRI) was attributed to his chronic constipation and straining. Labs showed improved hemoglobin levels at $10.3 \mathrm{~g} / \mathrm{dL}$, low iron saturation at $4 \%$, low iron levels at 20 $\mu \mathrm{g} / \mathrm{dL}$, elevated total iron binding capacity at $469 \mu \mathrm{g} / \mathrm{dL}$, and low serum ferritin at $7 \mathrm{ng} / \mathrm{mL}$. A diet history obtained at this point showed a very picky eater suggesting this was the more likely cause of anemia. He was started on oral omeprazole 40 $\mathrm{mg}$ for EOE, and a repeat endoscopy has been planned to see if his EOE is proton pump inhibitor responsive. He was continued on mesalamine suppository $1 \mathrm{~g}$ QD as needed and ferrous sulfate $324 \mathrm{mg}$ for his rectal ulcer and iron deficiency anemia. He was also started on oral docusate $200 \mathrm{mg}$ daily to help with regular bowel movements. At his 6-month follow-up visit, he reported being clinically asymptomatic and no longer had fecal impaction. His mesalamine suppository was stopped since he had been stooling daily with the docusate. Omeprazole 40 mg was continued and his repeat endoscopy has been scheduled 2 months from now. He will also have a repeat colonoscopy at the same time to follow-up on the solitary rectal ulcer.

\section{Discussion}

We present two pediatrics cases of SRUS whose symptoms present similar to that of IBD. These cases aforementioned differ from available literature such that case 2 presents a correct diagnosis of SRUS within 2 months of the initial presentation of symptoms, marking it well below the average time interval of 5 years between the onset of symptoms and implementing effective treatment in pediatrics populations with SRUS [4, 7]. Furthermore, both cases presented with elevated ESR but normal inflammatory markers such as fecal calprotectin and CRP. Although ESR was elevated, this is generally a non-specific inflammatory marker.

Though SRUS is well recognized in adults, this benign rectal disease is often initially misdiagnosed as malignancy or 
Table 1. Labs During Hospital Admission (Case 2)

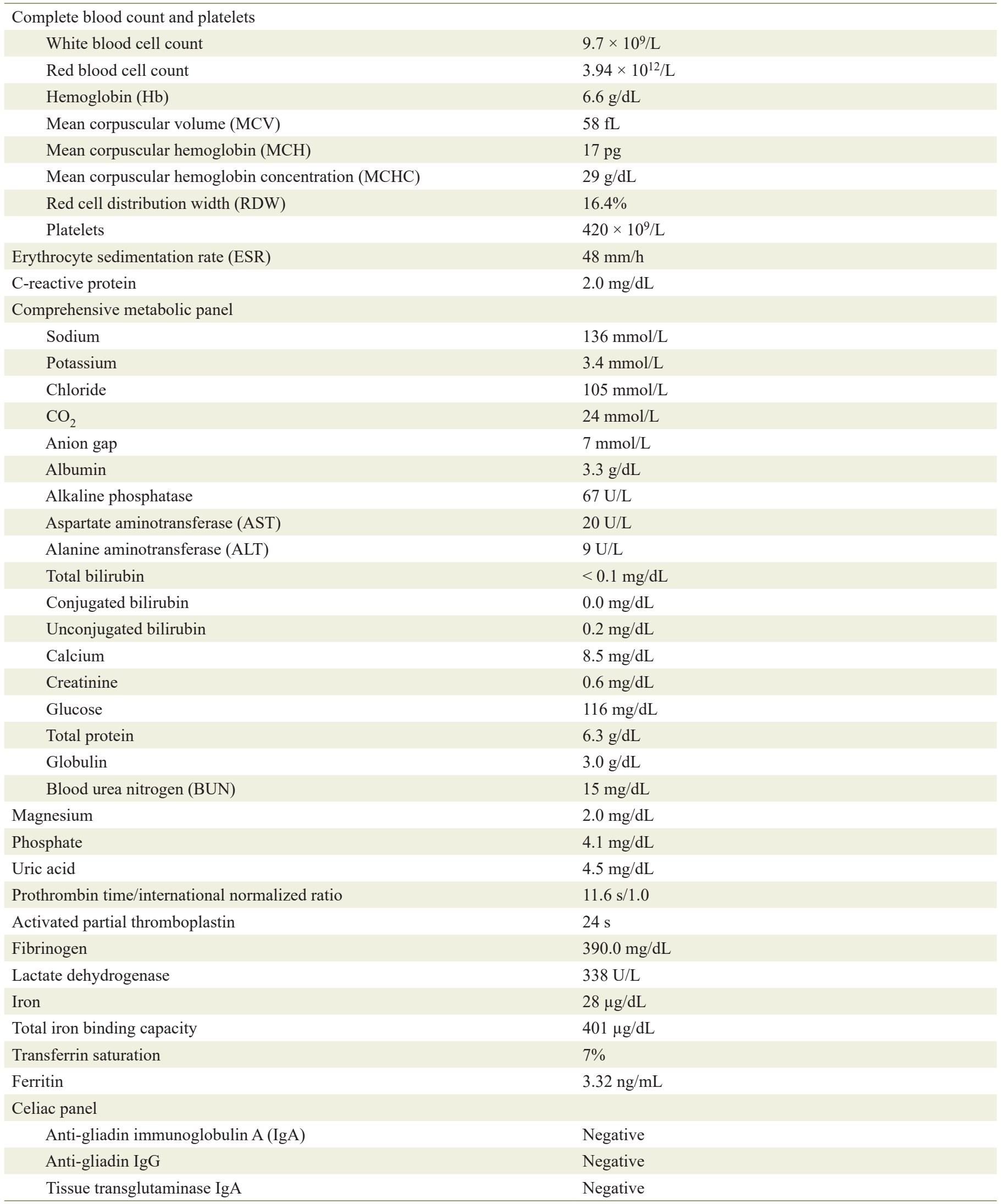


Table 1. Labs During Hospital Admission (Case 2) - (continued)

\begin{tabular}{|c|c|}
\hline Fecal occult blood test (FOBT) & Positive \\
\hline Fecal calprotectin & $<15.6 \mathrm{mg} / \mathrm{kg}$ \\
\hline \multicolumn{2}{|l|}{ Gastrointestinal panel } \\
\hline Clostridium difficile toxin A/B PCR, stool & Clostridium difficile toxin A/B gene PCR detected \\
\hline Clostridium difficile toxin A/B enzyme immunoassay (EIA), stool & Not detected \\
\hline Vibrio PCR, stool & Not detected \\
\hline Vibrio cholera PCR, stool & Not detected \\
\hline Yersinia enterocolitica PCR, stool & Not detected \\
\hline Enteroaggregative Escherichia coli (E. coli) PCR, stool & Not detected \\
\hline E. coli $0157 \mathrm{PCR}$, stool & Not detected \\
\hline Shigella species PCR, stool & Not detected \\
\hline Cryptosporidium PCR, stool & Not detected \\
\hline Cyclospora cayetanesis PCR, stool & Not detected \\
\hline Entamoeba histolytica PCR, stool & Not detected \\
\hline Giardia lamblia PCR, stool & Not detected \\
\hline Adenovirus F 40/41 PCR, stool & Not detected \\
\hline Astrovirus PCR, stool & Not detected \\
\hline Norovirus GI/GII PCR, stool & Not detected \\
\hline
\end{tabular}

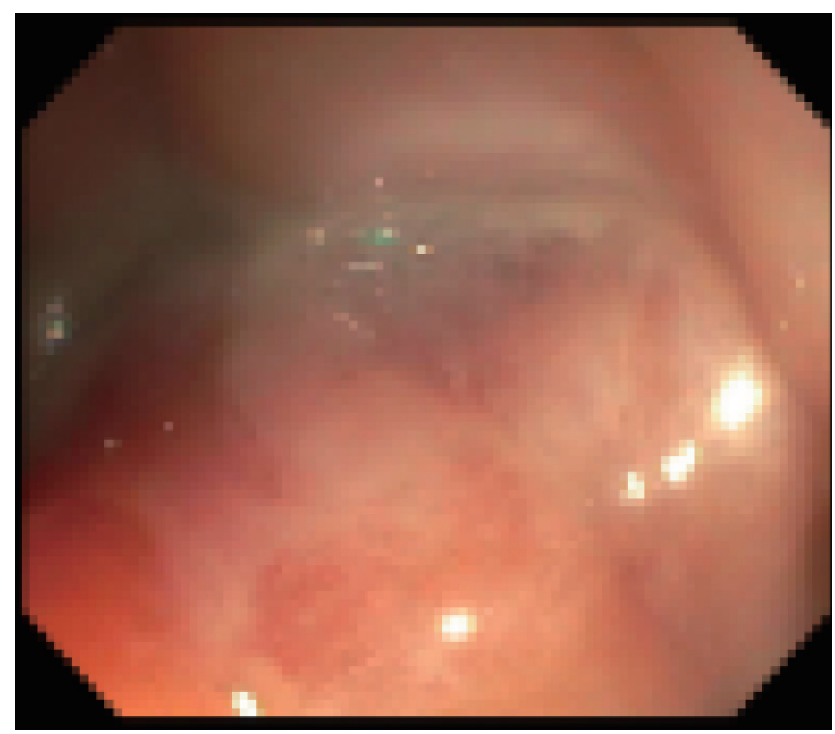

Figure 3. Colonoscopy results during hospital admission showing a localized area of moderately congested mucosa and exudate found in the rectum.
IBD in children $[4,5,7]$. Due to the fact that SRUS has various presentations and it is difficult to recognize both the macroscopic and histopathological changes during childhood, the time interval between the onset of symptoms and implementing effective treatment varies drastically. In adult populations, this interval can span between 3 months to 30 years, with the average being 5 years. In children, this interval ranges from 1.2 to 5.5 years, with an average of 5 years from onset of symptoms to diagnosis $[4,7]$. Of the limited pediatric SRUS cases reported in literature, a majority of the cases are found in children older than 8 years, with the youngest reported patient of 1.5 years $[7,8]$. In the cases presented, the 4-year-old male was correctly diagnosed 8 years after initial presentation. However, the second case was diagnosed within the first 2 months of initial presentation.

SRUS affects men and women equally. However, literature indicates a smaller predominance in women [5]. In children, SRUS is rare, and therefore, the prevalence is not known. However, in a large case series of Iranian children, 12 of 256 children (5\%) were diagnosed with SRUS [9]. The most common presenting symptoms include rectal bleeding, constipation, abdominal pain and alternating diarrhea. Endoscopy 
usually shows visible erythema or inflammation, particularly of the distal rectum. Furthermore, patients are often initially diagnosed with IBD and then subsequently diagnosed with SRUS [4]. The two cases we present adhere to this path of an initial diagnosis of IBD followed by a subsequent diagnosis of SRUS since both presented with symptoms very similar to that of IBD. SRUS can present with various clinical symptoms. The first case presented with rectal bleeding, constipation, and straining with defecation, with a workup revealing rectal ulcers and positive ANA. The second case presented with a history of chronic constipation and stool incontinence was found to have a very low hemoglobin level likely due to a poor diet.

Although the pathophysiology of SRUS is not well known, it is hypothesized that chronic damage to the mucosa from direct trauma or local ischemia caused by tenesmus, straining during constipation, intussusception of the rectal mucosa, and inflammation from hard stools may play a role [10]. Since both of our cases presented with straining with defecation and a history of chronic constipation, it is important to consider SRUS in the differential diagnosis.

Further investigation of symptoms, macroscopic findings and histopathologic reports suggest SRUS as a more accurate diagnosis. IBD and malignancy are differentiated from SRUS with histopathology findings. Some microscopic abnormalities of IBD are: crypt atrophy, which includes crypt shortening and widening of spaces between crypts, increased inflammation markers, and the presence of granulomas and mucin depletion [11]. Yet, SRUS follows the characteristic appearance of fibromuscular obliteration in the lamina propria, thickening of the mucosal layer, mucus proliferation and gland distortion and mild inflammation [9]. However, there are sometimes minor microscopic changes seen in SRUS such as surface erosion, reactive hyperplasia, mild inflammation, and distorted crypts that may lead to false diagnosis of IBD [5]. In the first case, colonoscopy revealed a single rectal ulcer and histology confirmed this solitary ulcer with necrotic exudate and acute and chronic inflammatory cells with atrophic, reactive mucosal glands. In the second case, colonoscopy showed wall thickening of the rectum, focal acute and chronic inflammation with erosions and ulcers. However, in both cases, there were no granulomas or dysplasia seen in histology and inflammatory markers such as fecal calprotectin, ESR, and CRP were all normal, thus supporting the benign nature of SRUS. Additionally, in both cases, biopsies taken from the transverse colon, cecum, and terminal ileum were all normal.

A conservative treatment plan for SRUS is directed towards patients with mild to moderate symptoms with no significant prolapse. It includes education of the patient to avoid straining, minimize the amount of time spent on the commode, and increased consumption of a high-fiber diet and bulk laxatives [12]. Furthermore, topical treatments such as sucralfate, salicylates, corticosteroids, sulfasalazine, mesalazine, and topical fibrin sealent have been shown to improve symptoms [13]. If symptoms persist, gut directed biofeedback or behavioral therapy can be used, which helps reduce excessive straining associated with defecating $[14,15]$. If neither a conservative method nor biofeedback is therapeutic, and if patients have a rectal prolapse or intussusception, surgical intervention may be considered such as ulcer excision or treatment of the rectal prolapse or rectal intus- susception [13]. If patients do not have a rectal prolapse or intussusception, but neither a conservative method nor biofeedback is therapeutic, local excision may be considered for symptomatic relief $[15,16]$. Both SRUS cases followed a conservative treatment plan where the former was treated with mesalamine, docusate, and increasing fiber intake. The latter was treated with mesalamine and docusate. Both treatments are ongoing and will be reassessed at the next follow-up visit.

\section{Learning points}

These cases highlight the difficulty and necessity of differentiating between IBD and SRUS. We emphasize the diagnosis of our 15-year-old patient made within 2 months since in identifying key findings of SRUS, the initial diagnosis of IBD was eliminated and the time interval between correct diagnosis and implementing effective treatment was shortened. The diagnosis of SRUS may require an extensive, holistic and well documented approach supported by both macroscopic and histopathological findings. It is important to keep SRUS high on the differential diagnoses when a pediatric patient presents with symptoms with that of IBD, as the treatment of IBD may not be useful for SRUS. Furthermore, correctly diagnosing SRUS can decrease the chances of unnecessary surgeries and increase the emotional stability promised by the reassurance of the benign nature of the disease. With this, additional studies of larger pediatric populations with SRUS and long-term follow-up are also necessary in creating reliable treatment protocols.

\section{Acknowledgments}

None to declare.

\section{Financial Disclosure}

The authors have no affiliations with or involvement in any organization or entity with any financial interest of non-financial interest in the subject matter or materials discussed in this manuscript.

\section{Conflict of Interest}

None to declare.

\section{Informed Consent}

Not applicable.

\section{Author Contributions}

Conceptualization: ST, PR, JT. Writing of original draft preparation: ST, PR, JT. Review and editing: ST, PR, JT. All authors 
have read and agreed to the published version of the manuscript.

\section{Data Availability}

The authors declare that data supporting the findings of this study are available within this article.

\section{References}

1. Thirumal P, Sumathi B, Nirmala D. A clinical entity often missed-solitary rectal ulcer syndrome in children. Front Pediatr. 2020;8:396.

2. Martin CJ, Parks TG, Biggart JD. Solitary rectal ulcer syndrome in Northern Ireland. 1971-1980. Br J Surg. 1981; 68(10):744-747.

3. Tjandra JJ, Fazio VW, Petras RE, Lavery IC, Oakley JR, Milsom JW, Church JM. Clinical and pathologic factors associated with delayed diagnosis in solitary rectal ulcer syndrome. Dis Colon Rectum. 1993;36(2):146-153.

4. Perito ER, Mileti E, Dalal DH, Cho SJ, Ferrell LD, McCracken M, Heyman MB. Solitary rectal ulcer syndrome in children and adolescents. J Pediatr Gastroenterol Nutr. 2012;54(2):266-270.

5. Zhu QC, Shen RR, Qin HL, Wang Y. Solitary rectal ulcer syndrome: clinical features, pathophysiology, diagnosis and treatment strategies. World J Gastroenterol. 2014;20(3):738-744.

6. Abreu M, Azevedo Alves R, Pinto J, Campos M, Aroso S. Solitary rectal ulcer syndrome: a paediatric case report. GE Port J Gastroenterol. 2017;24(3):142-146.

7. Dehghani SM, Malekpour A, Haghighat M. Solitary rectal ulcer syndrome in children: a literature review. World J Gastroenterol. 2012;18(45):6541-6545.

8. Suresh N, Ganesh R, Sathiyasekaran M. Solitary rectal ulcer syndrome: a case series. Indian Pediatr. 2010;47(12):10591061.

9. Forootan M, Darvishi M. Solitary rectal ulcer syndrome: A systematic review. Medicine (Baltimore). 2018;97(18): e0565.

10. Dehghani SM, Haghighat M, Imanieh MH, Geramizadeh B. Solitary rectal ulcer syndrome in children: a prospective study of cases from southern Iran. Eur J Gastroenterol Hepatol. 2008;20(2):93-95.

11. Feakins RM, British Society of G. Inflammatory bowel disease biopsies: updated British Society of Gastroenterology reporting guidelines. J Clin Pathol. 2013; 66(12):1005-1026.

12. Poddar U, Yachha SK, Krishnani N, Kumari N, Srivastava A, Sen Sarma M. Solitary rectal ulcer syndrome in children: a report of 140 cases. J Pediatr Gastroenterol Nutr. 2020;71(1):29-33.

13. Edden Y, Shih SS, Wexner SD. Solitary rectal ulcer syndrome and stercoral ulcers. Gastroenterol Clin North Am. 2009;38(3):541-545.

14. Vaizey CJ, Roy AJ, Kamm MA. Prospective evaluation of the treatment of solitary rectal ulcer syndrome with biofeedback. Gut. 1997;41(6):817-820.

15. Niv Y, Bat L. Solitary rectal ulcer syndrome - clinical, endoscopic, and histological spectrum. Am J Gastroenterol. 1986;81(6):486-491.

16. Kowalska-Duplaga K, Lazowska-Przeorek I, Karolewska-Bochenek K, Woynarowski M, Czaja-Bulsa G, Stawarski A, Pieczarkowski S, et al. Solitary rectal ulcer syndrome in children: a case series study. Adv Exp Med Biol. 2017;1020:105-112. 